\title{
Crack Detection by Optical Voice Recorder Based on Digital Holography
}

\author{
Gülhan USTABAŞ KAYA and Zehra SARAÇ* \\ Zonguldak Bülent Ecevit University, Faculty of Engineering, Department of Electrical and Electronics Engineering, \\ Incivez, Zonguldak 67100, Turkey \\ ${ }^{*}$ Corresponding author: Zehra SARAÇ_Ｅ-mail: zehrasarac@beun.edu.tr
}

\begin{abstract}
The detection of crack on materials is an important issue in industry. On the contrary to conventional methods, such as manual inspection, sensor detection, and image processing techniques, a new simple method to detect the crack is proposed with optical voice recorder based on digital holography in this paper. Holograms obtained with sound waves passing through the materials are recorded by using the digital holography technique. Temporal behavior of the sound wave passing through the material, which is obtained from these holograms, gives image of crack. In this article, cracks in various materials are determined by the proposed new method, and crack images obtained with this new system are presented.
\end{abstract}

Keywords: Crack detection; sound wave; digital holography; material surface

Citation: Gülhan USTABAŞ KAYA and Zehra SARAÇ, "Crack Detection by Optical Voice Recorder Based on Digital Holography," Photonic Sensors, 2019, 9(4): 327-336.

\section{Introduction}

The deformations (defects), which frequently occur in the material, have been investigated with a variety of studies. These deformations are porosity, debonding, delamination, crack, etc. From among these, the detection of crack is one of the main issues for researchers [1-6]. It can be made in two ways: destructive testing and non-destructive testing [7-10]. In destructive testing, since manual inspection depends on specialist's experience with the help of surveying instruments, the accuracy cannot be guaranteed in quantitative analysis. On the other hand, for reliable and fast crack surface analysis, automatic crack detection is developed instead of the manual inspection procedures. This detection method is very effective for some of the non-destructive testing techniques, such as infrared and thermal testing, ultrasonic testing, laser testing, radiographic testing, and testing with image processing [11-22]. Moreover, in destructive testing, due to the fact that the surface texture has been destroyed in destructive testing, non-destructive testing techniques are preferred to detect the crack in the materials.

For non-destructive testing (NDT), there are some optical methods, which use the non-contact measurement system such as interferometry and holograph [23, 24]. Among these techniques, digital holography (DH) is commonly used to measure deformations [25-33], and at the same time it is used to determine the properties of material. For example, Şan et al. [34] tested the performance of core materials under the effect of static magnetic field by using DH. Furthermore, DH technique is also used to test the surface flatness and detect

Received: 21 November 2018 / Revised: 16 January 2019

(C) The Author(s) 2019. This article is published with open access at Springerlink.com

DOI: $10.1007 / \mathrm{s} 13320-019-0541-1$

Article type: Regular 
cracks in material [35-37].

In this work, such a system based on off-axis digital holography, where the acusto-optics effect is formed, is used to detect crack. This system was recently developed by Matoba et al. [38]. The crack detection with this system can be made by using frequency or intensity distributions of sound wave reconstructed from sound holograms [39]. In this paper, the latter is used for crack detection. How the sound wave behaves when passing through the material can be determined by using the acusto-optics effect. In this context, for the first time, the temporal phase profiles (sound wave) obtained from the sound holograms are used to measure this effect. For each pixel point of recorded holograms, the temporal phase profile is extracted and 3-dimensional (3D) images are obtained by using the maximum amplitude values in these profiles. Since the amplitude change is expected in the fractured regions, the presence and shape of the crack are determined by analyzing the 3D images after unwrapping process of the maximum amplitude values of temporal phase distributions. In this study, three different materials, namely, glass, wood, and metal, are investigated. Instead of using more than one specimen, it is intended to give results for different materials. Thus, it is proved that no matter which material is tested with the proposed system, cracks can be determined. In addition, the change of the sound wave around the crack in the material over time can be visualized with the proposed system. Lastly, it is concluded that the intended system can indicates if the specimen have crack or not.

The rest of the paper is organized as follows. In Section 2, the methodology is introduced. The method used for reconstruction of sound waves is given in this section. The experimental results obtained to show cracks in materials are presented in Section 3. The last section is defined as conclusion part.

\section{Methodology}

The purpose of this paper is to detect cracks on material and to image these cracks by NDT. For this, an off axis-digital holographic interferometry (see Fig. 1) [39] is used and holograms obtained with the sound waves passing through the material on object arm of this interferometry are recorded. In this interferometry, He-Ne laser is selected as a light source with the output power of $10 \mathrm{~mW}$ and wavelength of $633 \mathrm{~nm}$.

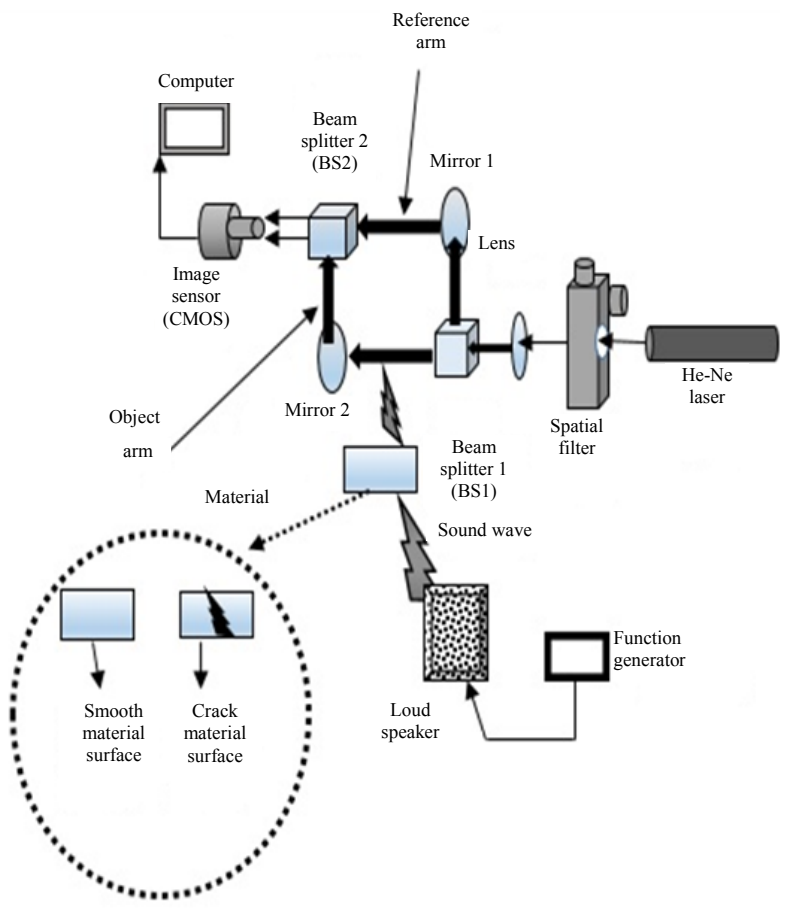

Fig. 1 Optical setup with schematic diagram [39].

A beam from He-Ne laser is filtered by using spatial filter and expanded by using lens. Then, the expanded light is split into two rays by using a cube beam splitter (BS1) to form the reference and object arms. BS1 has a division ratio of $50: 50$. The reference beam is directed to Mirror 1 (M1), and the object beam is directed to Mirror 2 (M2). Then, these beams are sent to the beam splitter (BS2) and complementary metal oxide semiconductor (CMOS) camera, respectively. To create the phase difference between two arms, the object beam is modulated with the sound wave. The sound wave produced by using function generator is transmitted to medium 
by a loudspeaker. After that, it is propagated through the material surface.

Object and reference beams are combined to create a hologram on the CMOS camera $(132 \times 132$ pixels), whose pixel size is $14 \mu \mathrm{m} \times 14 \mu \mathrm{m}$.

In this system, it is possible to detect the presence of a crack in two ways. The first way is based on the examination of a shift at frequency of the sound wave reconstructed from digital holograms [39]. The second way is based on the intensity of voice passing through the material.

In current study, how the sound wave behaves when passing through the material is presented by extracting the 3 dimensional (3D) image. Thus, the crack detection is performed by determining which points of the material have high or low amplitude while the sound wave passes through the material. In this context, the sound waves transmitted to the medium are passed through a material and transferred to the object arm of the interferometer. Thus, the sound holograms are recorded. Firstly, temporal phase profiles are found for each pixel point of the sound holograms. The maximum amplitude values of these phase profiles obtained by using $N$ holograms (6000 holograms in this work) are determined. So, the maximum amplitude value as much as the pixel number of a hologram is obtained. As a result, the behavior of the sound wave passing through the material is given in 3D. The amplitude values of the sound waves passing through the region where the crack is presented are displayed with the 3D images. The steps of this crack detection are presented by the flowchart in Fig. 2 .

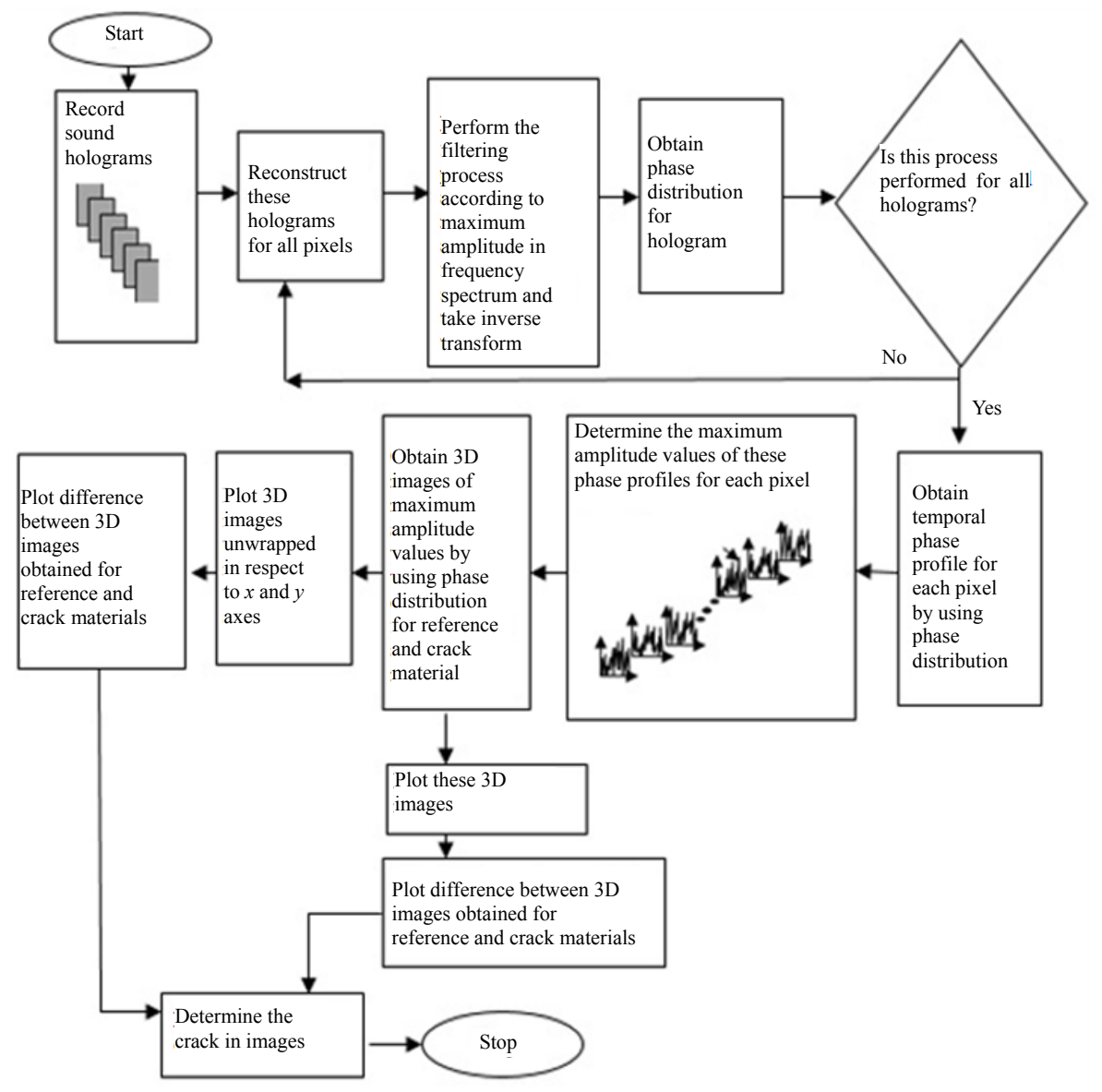

Fig. 2 Flowchart of crack detection.

In this study, the temporal phase information is obtained for all pixels. This information is calculated by using the Fourier transform method (FTM), which was proposed by Takeda [40] for the 
first time in 1982. The mathematical formula of recorded hologram can be expressed as follows:

$$
\begin{gathered}
I(x, y)=\left|A_{\mathrm{OB}}(x, y)\right|^{2}+\left|A_{\mathrm{REF}}(x, y)\right|^{2}+ \\
2\left|A_{\mathrm{OB}}(x, y)\right|\left|A_{\mathrm{REF}}(x, y)\right| \cos (\Delta \theta) \\
\Delta \theta(x, y)=k z+\varphi(x, y)=\left(\frac{2 \pi}{\lambda}\right)(n-1)\left(\frac{P}{P_{0}}-1\right)
\end{gathered}
$$

where the complex fields of object and reference waves are expressed as $A_{\mathrm{OB}}(x, y)$ and $A_{\mathrm{REF}}(x, y)$. The coordinate of hologram plane is given by $(x, y)$. In addition, the phase of hologram is expressed as $\Delta \theta(x, y)$. The atmospheric pressure and the pressure due to the sound waves are defined as $P_{0}$ and $P$, respectively. The refractive index of air is given with $n$. The temporal phase is formed by this refractive index change. Moreover, in the expression $I(x, y)$, the first two terms on the right side of the equation contain completely the amplitude information of the hologram, while the third term includes the phase information.

The Fourier transform is performed in order to extract the temporal phase distribution from the series of hologram, and it is expressed as follows:

$$
G\left(k_{x, y}\right)=\mathfrak{I}\{I(x, y)\} .
$$

The first step of signal processing is to take the line by line Fourier transform of the hologram. Then the direct current (DC) component and the imaginary part are eliminated by band pass filtering process. Finally, the phase information [as expressed in (4)] is obtained by taking the inverse fast Fourier transform (IFFT).

$$
\begin{gathered}
c(x, y)=\mathfrak{J}^{-1}\left\{G\left(k_{x, y}\right)\right\} \\
\varphi(x, y)=\tan ^{-1} \frac{\operatorname{Im}\{c(x, y)\}}{\operatorname{Re}\{c(x, y)\}}
\end{gathered}
$$

where $\varphi(x, y)$ is a wrap phase and in the interval of $(-\pi,+\pi)$. This phase is unwrapped by a suitable algorithm, which is presented in MATLAB, in respect to $x$ and $y$ axes.

\section{Results and discussion}

In the proposed system, crack detection is performed by imaging sound wave passing through the material with digital holographic interferometry. Crack analysis is carried out in two steps. In Step 1, the glass and wooden materials, on which crack can be seen with naked eye, are used. In Step 2, measurements are taken from the metal surface, on which crack cannot be seen. Sound waves can be used at different frequencies for crack detection in the materials used in this study. These sound waves are passed through the surface by means of a loudspeaker and transferred to the object arm in the interferometric system and the sound holograms are recorded.

As the sound wave passes through these materials, its amplitude changes over time. Shortly, in this state, while the sound wave passes through a material, its energy changes. Because the atoms in the material are in motion, the direction of the sound wave moving in the material changes. This means that the frequency and the wavelength of the sound also change. If there is a crack in the material, the incoming sound wave will be subject to diffraction and scattering. With this scattering, the amplitude of the sound reduces in the cracked region. Therefore, the propagation speed of the sound wave decreases and the amplitude (intensity-energy) of the wave also reduces accordingly. In this study, the difference between the reference (sound wave passing through non-cracked surface) and wave passing through the cracked surface is given by $3 \mathrm{D}$ images. Whether the material is cracked or not is determined by looking at these 3D images.

The crack detection with the proposed system is firstly made for material with visible cracks. For example, it is possible to see the cracks found on glass and wooden surfaces. In the first phase of the study, the crack detection is performed by using reference and cracked glass surfaces, respectively. Sound wave, which has a frequency value of $1 \mathrm{kHz}$, is used to determine the crack. In digital holographic interferometry, when sound hologram is recorded, the sampling is made according to Nyquist criteria for reconstruction of sound wave. That is, the 
sampling frequency should be at least 2 times or greater than the maximum frequency of the used sound wave. If the sampling frequency of the sound wave is $1 \mathrm{fmax}$, the recording time of the imaging sensor must be shorter than 1/2 fmax. Therefore, a minimum of 2000 samples per second should be taken for a sound wave with a frequency of $1 \mathrm{kHz}$. The high-speed CMOS camera, which is used as an imaging sensor in the experimental setup, has a high resolution. If the used sampling frequency is increased, the resolution of this camera will be reduced. Reduction of resolution results in the distortion of 3D images obtained for the crack. In this case, the crack detection cannot be done correctly. In some studies, the results are also obtained for sound waves with a frequency higher than $1 \mathrm{kHz}$. However, due to the above-mentioned reason, the cracks in the images could not be determined exactly. Therefore, a sound wave with a

\section{frequency of $1 \mathrm{kHz}$ is used.}

The reference and cracked glass surfaces used in Fig. 3 are given. Figure 3(a) shows the reference glass surface and Fig. 3(b) shows the cracked glass surface. This crack is shown by a white circle in Fig. 3(b).

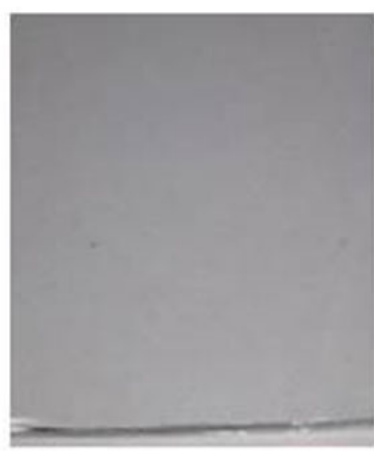

(a)

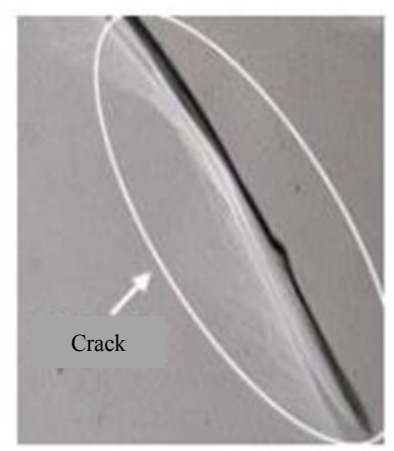

(b)
Fig. 3 Glass materials (a) reference glass surface and (b) glass surface with crack.

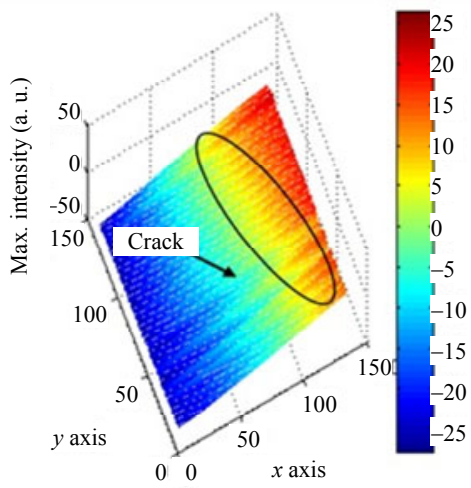

(b)

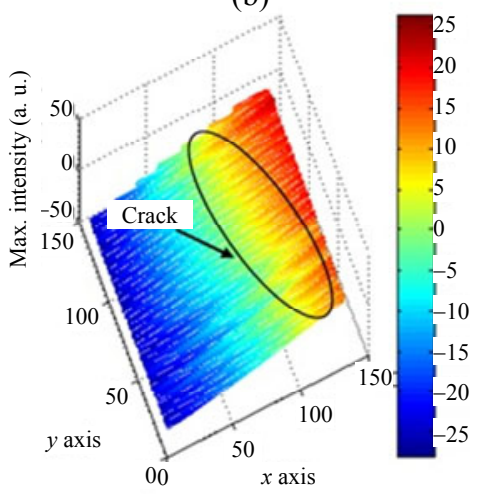

(d)

Fig. 4 3D image of the temporal behavior of the sound wave passing through the glass surface for :(a) unwrapped situation of maximum values of temporal phase distribution for reference glass material, (b) unwrapped situation of maximum values of temporal phase distribution for crack glass material, (c) wrapped state of the difference between (a) and (b), and (d) unwrapped situation of the difference between (a) and (b). 
In Fig. 4, 3D image is presented to show the temporal behavior of the sound wave passing through the reference and cracked glass surfaces. Whereas the sound wave passes directly from the non-cracked section, it passes through the cracked part by decreasing its intensity. This means that the intensity of the sound wave passing through the cracking zone is decreasing, which can be clearly seen in Fig. 4. The crack on the figure is indicated by a black circle.

In Fig. 4, the $x$ and $y$ axes represent the pixel numbers of the hologram. The $z$-axis refers to the intensity (maximum amplitude value) of the sound wave after passing through the material. The unwrapped situation of the maximum values of temporal phase distributions for reference glass material is shown in Fig. 4(a). Likewise, this distribution is presented in Fig. 4(b) for the crack glass material. In addition, the difference between reference and crack glass materials is obtained for both wrapped and unwrapped situations. They are also shown in Figs. 4(c) and 4(d), respectively.

However, as the sound wave passes through the cracked glass surface, its intensity decreases. This state can be seen from Fig. 4(c) and 4(d).

3D image obtained for the glass surface clearly shows that crack detection by using sound wave in the digital holography system is a very convenient method for non-destructive measurement. When Fig. 4(d) and Fig.3(b) are compared, it can be clearly seen that the crack detection on the glass surface is accurately performed.

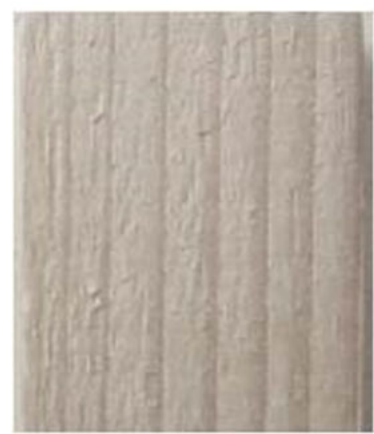

(a)

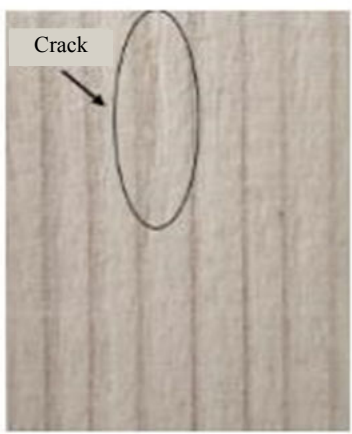

(b)
Fig. 5 Wooden materials: (a) reference wooden surface and (b) wooden surface with crack.
In the second phase of this study, crack detection is performed for wooden materials. In Fig. 5, reference and cracked wooden materials are shown. The crack in wooden materials is shown with a black circle in Fig. 5(b). In this part of the work, the sound wave with a frequency value of $1 \mathrm{kHz}$ is used when recording hologram. This sound wave is transmitted to the object arm of interferometer when passing through the reference and cracked materials, respectively.

As seen from Fig. 5(b), the crack is located at the upper right side of wooden materials. In the 3D image shown in Fig. 6, the temporal behavior of the sound wave as it passes through the reference and cracked wooden materials is presented. From this $3 \mathrm{D}$ image, the crack is clearly visible.

In Figs. 6(a) and 6(b), the unwrapped situations of the maximum values of temporal phase distributions for reference and cracked wooden materials are given, respectively. On the other hand, the difference between reference and cracked wooden materials, which are obtained under both wrapped and unwrapped situations respectively, is presented in Figs. 6(c) and 6(d). When Figs. 6(c), 6(d), and 5(b) are compared, cracks in wooden materials can be seen. As in the glass material, when the sound wave passes through the cracked wooden materials, the sound intensity decreases. As it can be seen from Fig. 6(b), there is a significant drop in intensity where the crack occurs. This means that when the sound wave passes through the cracked area, the intensity of the sound is reduced. On the other hand, the intensity of the sound passing through the non-cracked regions has a high amplitude value. Therefore, a bump is observed in cracked area, which can be seen clearly in Figs. 6(c) and 6(d). This bump is shown in black circle.

In order to prove the validity of this work, first, the existing crack on the material that can be easily seen with the naked eye is determined (glass and wooden materials). In the light of the results obtained from system, the proposed system can be used for crack detection by non-destructive 
inspection. Furthermore, it is possible to detect cracks that are not visible through this system as well. For example, it is impossible to see cracks in metal materials by naked eyes. That's why, in the

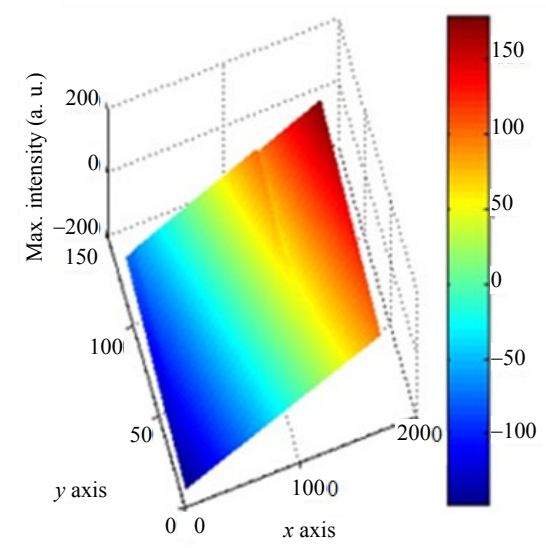

(a)

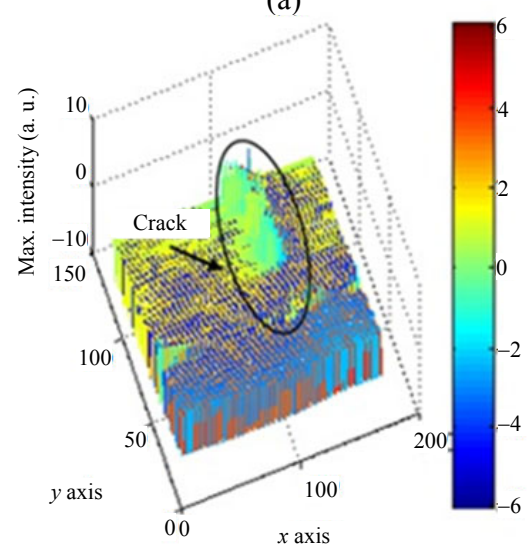

(c) third phase of this work, cracks on metal that are not seen by the naked eye are detected. Reference and cracked metal materials used in this study are shown in Fig. 7.

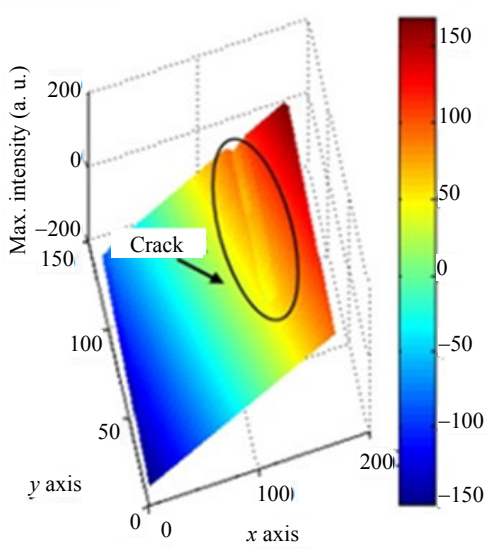

(b)

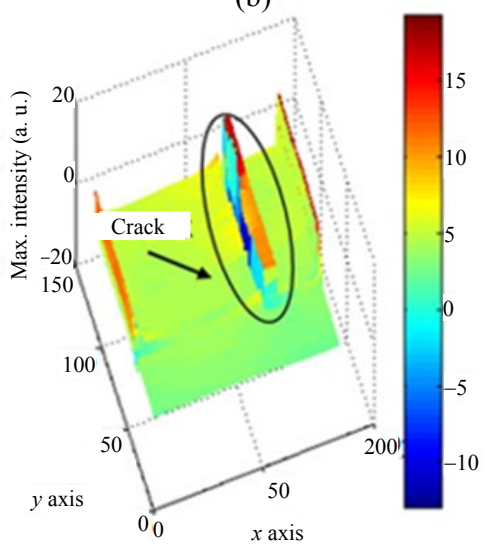

(d)

Fig. 6 3D image of the temporal behavior of the sound wave passing through the wooden surface for: (a) unwrapped situation of maximum values of temporal phase distribution for reference wooden material, (b) unwrapped situation of maximum values of temporal phase distribution for crack wooden material, (c) wrapped state of difference between (a) and (b), and (d) unwrapped situation of difference between (a) and (b).

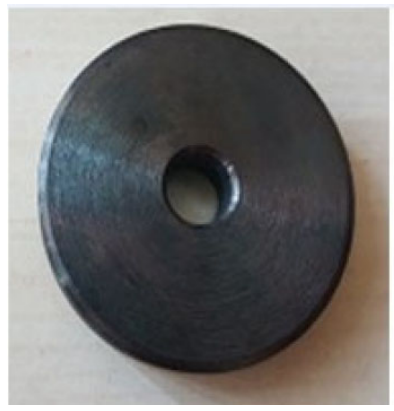

(a)

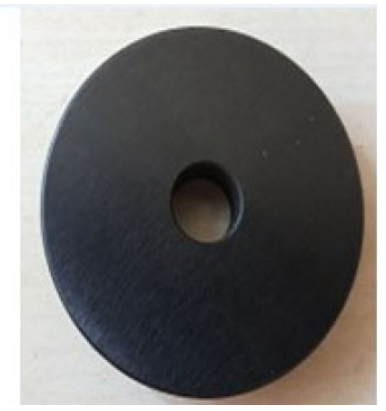

(b)

Fig. 7 Metal materials: (a) reference metal surface and (b) metal surface with crack.

Metals are damaged over time. These materials can be scratched, cracked, etc. If the crack size is in millimeter, it is possible to see the cracks on the

surface of the material. In this case, there is no need for a system to detect cracks. If the size of these cracks is at the micrometer level, cracks cannot be seen by naked eyes. In this case, a device or a system for crack detection must be used. The cracks on the glass surfaces can be seen easily because they are in millimeters. Since it is impossible to see the cracks in the micrometer level in metals, crack detection has been done with the proposed system in this study. In this article, in order to increase the effect of the study, both the visible and invisible cracks for the naked eyes are imaged.

Since cracks in metal materials cannot be seen, 
the image of the crack is given by scanning electron microscope (SEM). In the SEM image, the crack is marked with a white circle in Fig. 8. The crack in this image resembles the veins of a leaf.

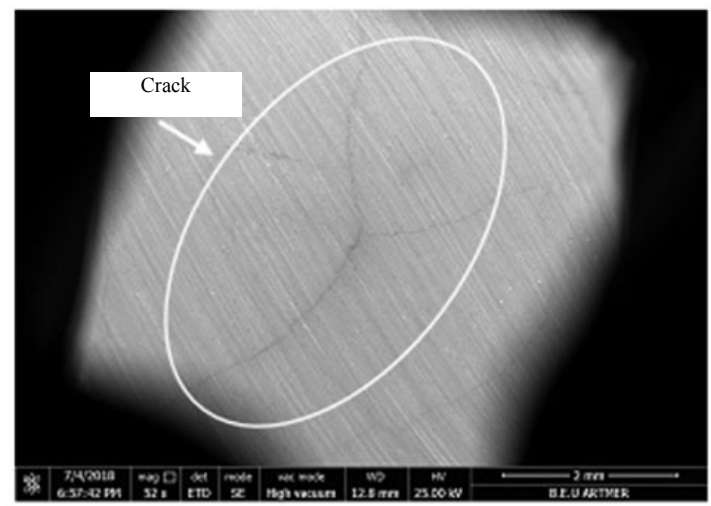

Fig. 8 SEM image of metal surface with crack.

As mentioned above, it is possible to detect cracks with holographic recording of sound waves

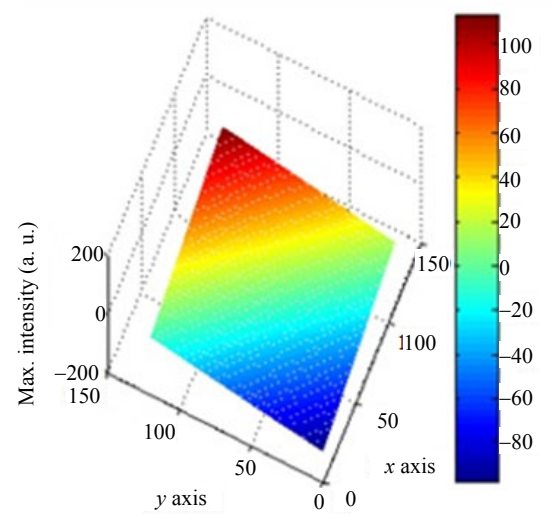

(a)

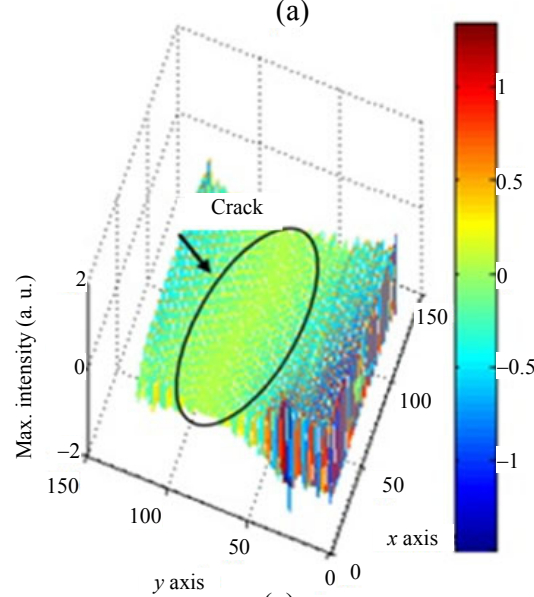

(c) passing through metal material. This is done by extracting a 3D image showing how the intensity changes over time as the sound wave passes through the material, as shown in Fig. 9. Here, the sound wave with a frequency of $2 \mathrm{kHz}$ is used when recording hologram. This sound wave is given to the object arm of interferometer by being passed through the reference and cracked materials, respectively.

The unwrapped situations of the maximum values of temporal phase distributions for reference and cracked metal material are shown in Figs.9(a) and 9(b), respectively. Moreover, the differences between both reference and crack metal materials are obtained for wrapped and unwrapped situations, which are presented in Figs. 9(c) and 9(d), respectively.

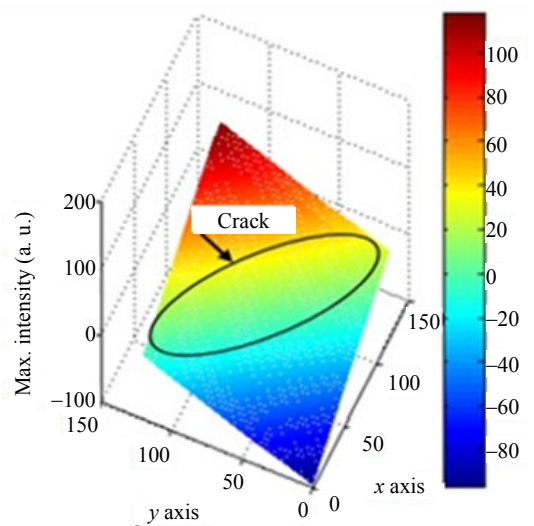

(b)

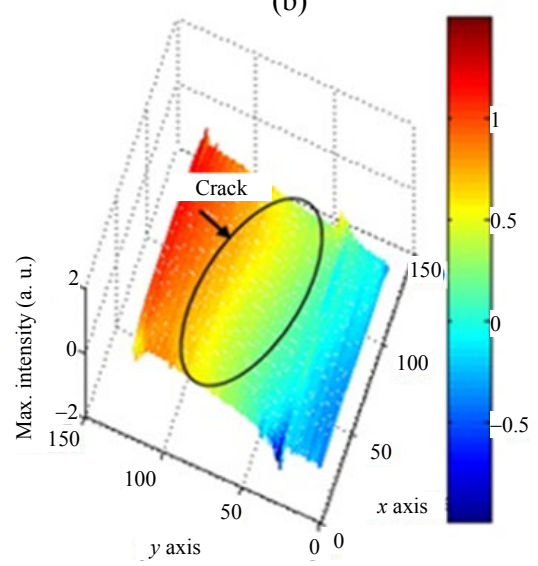

(d)

Fig. 9 3D image of the temporal behavior of the sound wave passing through the metal surface for: (a) unwrapped situation of maximum values of temporal phase distribution for reference metal material, (b) unwrapped situation of maximum values of temporal phase distribution for crack metal material, (c) wrapped state of difference between (a) and (b), and (d) unwrapped situation of difference between (a) and (b). 
In Fig. 9, cracks in the metal material can easily be discerned. The crack portion of metal materials is shown by a black circle in Figs. 9(b) to 9(d). When the sound wave passes through the crack zone, its intensity drops, which is illustrated in green color. 3D images in Figs. 9(c) and 9(d) show that the crack pattern obtained by the proposed system is very similar to the crack pattern given by SEM, which means that it is possible to detect cracks whatever they can be seen or not by naked eyes.

\section{Conclusions}

In this paper, it is shown that it is feasible to detect cracks on materials by using sound holograms, which are recorded with digital holography. The recorded sound holograms are digitally analyzed to extract temporal amplitude distribution (sound wave, which is transferred to medium while the hologram is recorded in holographic setup). In addition, 3D images of sound wave whose behavior changes when passing through material are given and the crack detection is done by using $3 \mathrm{D}$ images.

Although the crack detection can be performed for glass, wooden and metal materials in this study, the amount of the crack cannot be given. In the future study, it is planned to give the size of the cracks with the system proposed in this study. As a result, this study is thought to bring a new perspective on the issue of the contactless crack detection.

\section{Acknowledgment}

We acknowledge the director of research and development of UÇAR Metal Company who contributed towards the article. He provided material for test measurements.

This study was funded by Department of Scientific Research Projects in Zonguldak Bulent Ecevit University (Grant No. 2016-75737790-04); Scientific and Technological Research Council of Turkey (TUBITAK) (Grant No. 116E303).

Open Access This article is distributed under the terms of the Creative Commons Attribution 4.0 International
License (http://creativecommons.org/licenses/by/4.0/), which permits unrestricted use, distribution, and reproduction in any medium, provided you give appropriate credit to the original author(s) and the source, provide a link to the Creative Commons license, and indicate if changes were made.

\section{References}

[1] Y. Zou, L. Tong, and G. P. Steven, "Vibration based model dependent damage (delamination) identification and health monitoring for composite structures-a review," Journal of Sound and Vibration, 2000, 230(2): 357-378.

[2] A. G. Mamalis, G. L. Petrossian, and D. E. Manolakos, "Effect of porosity and micro-defects on plastically deformed porous materials," Journal of Materials Processing Technology, 1999, 96(1-3): 117-123.

[3] Z. Xu, X. Fan, W. Zhang, and T. J. Wang, "Numerical analysis of anisotropic elasto-plastic deformation of porous materials with arbitrarily shaped pores," International Journal of Mechanical Sciences, 2015, 96-97(1): 121-131.

[4] T. Y. Jiang, Q. Z. Kong, D. Patil, Z. T. Luo, L. S. Huo, and G. B. Song, "Detection of debonding between FRP rebar and concrete structure using piezoceramic transducers and wavelet packet analysis," IEEE Sensors Journal, 2017, 17(7): 1992-1998.

[5] P. Liu, R. M. Groves, and R. Benedictus, “3D monitoring of delamination growth in a wind turbine blade composite using optical coherence tomography," NDT \& E International, 2014, 64(1): 52-58.

[6] A. Mohan and S. Poobal, "Crack detection using image processing: a critical review and analysis," Alexandria Engineering Journal, 2017, 57(1): 787-798.

[7] K. Tai, "The application of digital image processing technology in glass bottle crack detection system," Acta Technica, 2017, 62(1A): 381-390.

[8] A. M. Albishi and O. M. Ramahi, "Surface crack detection in metallic materials using sensitive microwave-based sensors," in Proceeding of 2016 IEEE 17th Annual Wireless and Microwave Technology Conference (WAMICON), Clearwater, FL, USA, 2016, pp. 1-3.

[9] Uçar metal Home Page. http://www.ucarmetal.com/ catlak-kontrol-cihazi.asp (Accessed June 13, 2018).

[10] W. Sudatham, H. Matsumoto, S. Takahashi, and K. Takamasu, "Non-contact measurement technique for dimensional metrology using optical comb," Measurement, 2016, 78(1): 381-387.

[11] S. Gholizadeh, "A review of non-destructive testing methods of composite materials," Procedia Structural Integrity, 2016, 1(1): 50-57.

[12] A. Katunin, K. Dragan, and M. Dziendzikowski, "Damage identification in aircraft composite structures: a case study using various nondestructive 
testing techniques," Composite Structures, 2015, 127(1): 1-9.

[13] P. Broberg, "Surface crack detection in welds using thermography," NDT\&E International, 2013, 57(1): 69-73.

[14] A. Keshtgar and M. Modarres, "Detecting crack initiation based on acoustic emission," Chemical Engineering Transactions, 2013, 33(1): 547-552.

[15] M. Aoyagi, T. Hiraguri, and T. Ueno, "Nondestructive detection of cracks near the surface of wooden boards by dynamic heat dissipation," Wood Science and Technology, 2014, 48(4): 773-786.

[16] J. Zhang, B. Huang, G. Zhang, and G. Y. Tian, "Wireless passive ultrahigh frequency RFID antenna sensor for surface crack monitoring and quantitative analysis," Sensors, 2018, 18(7): 2130-1-2130-11.

[17] D. Yu, S. Wenbin, Y. Hong, and Y. Yan, "Measurements of the characteristics of transparent material using digital holography," Advances in Materials Science and Engineering, 2013, 598737(1): $1-7$.

[18] P. A. Doyle and C. M. Scala, "Crack depth measurement by ultrasonics: a review," Ultrasonics, 1978, 16(4): 164-170.

[19] M. R. Martín, S. Lagüela, D. G. Aguilera, and J. Martínez, "Thermographic test for the geometric characterization of cracks in welding using IR image rectification," Automation in Construction, 2016, 61(1): 58-65.

[20] S. Dorafshan, M. Maguire, and W. Collins, "Infrared thermography for weld inspection: feasibility and application," Infrastructures, 2018, 3(4): 45-1-45-17.

[21] P. Prasanna, K. Dana, N. Gucunski, and B. Basily, "Computer-vision based crack detection and analysis," SPIE, 2012, 8345: 834542-1-834542-6.

[22] Z. Q. Shi, X. Y. Xu, J. J. Ma, D. Zhen, and H. Zhang, "Quantitative detection of cracks in steel using eddy current pulsed thermography," Sensors, 2018, 18(4): 1070-1-1070-14.

[23] U. Schnars and W. Juptner, Digital holography: digital hologram recording, numerical reconstruction and related techniques. Berlin, Germany: Springer-Verlag, 200:1-164.

[24] L. Yaroslavsky, Digital holography and digital image processing: principles, methods, algorithms. New York, USA: Springer US Publisher, 2004: $1-584$.

[25] S. Seebacher, W. Osten, P .Werner, and O. Jueptner, "Measuring shape and deformation of small objects using digital holography," SPIE, 1998, 3479: 104-115.

[26] I. Takahashi, T. Nomura, Y. Morimoto, S. Yoneyama, and M. Fujigaki, "Deformation measurement by digital holographic interferometry," SPIE, 2003, 5264: 206-213.

[27] W. Zhou, J. Peng, and Y. J. Yu, "Deformation measurement via digital holography," Guangxue
Jingmi Gongcheng/Optics and Precision Engineering, 2005, 13(1): 46-51.

[28] Y. Morimoto, T. Nomura, M. Fujigaki, S. Yoneyama, and I. Takahashi, "Deformation measurement by phase-shifting digital holographic interferometry," Experimental Mechanics, 2005, 45(1): 65-70.

[29] V. Lédl, F. Kaván, O. Matousek, R. Dolecek, and P. Psota, "Large displacement and deformation measurement by frequency sweeping digital holography," SPIE, 2017, 10603: 106030C-1106030C-7.

[30] D. D. Aguayo, F. M. Santoyo, M. H. De la Torre-I, M. D. S. Araiza, C. C. Mendez, and D. A. G. Hernandez, "Insect wing deformation measurements using high speed digital holographic interferometry," Optics Express, 2010, 18(6): 5661-5667.

[31] D. Claus, "High resolution digital holographic synthetic aperture applied to deformation measurement and extended depth of field method," Applied Optics, 2010, 49(16): 3187-3198.

[32] I. Yamaguchi, "Image formation and measurement of surface shape and deformation by phase-shifting digital holography," SPIE, 2005, 5642(1): 66-77.

[33] M. Dekiff, P. Berssenbrügge, B. Kemper, C. Denz, and D. Dirksen, "Simultaneous acquisition of 3D shape and deformation by combination of interferometric and correlation-based laser speckle metrology," Biomedical Optics Express, 2015, 6(12): 4825-4840.

[34] U. Şan, G. U. Kaya, and Z. Saraç, "Non-contact performance analysis of the core materials by digital holographic interferometry," IET Science, Measurement \& Technology, 2016, 10(1): 38-43.

[35] N. F. A. Maaboud, M. S. E. Bahrawi, and F. A. Aziz, "Digital holography in flatness and crack investigation," Metrology and Measurement Systems, 2010, 17(4): 583-588.

[36] P. Tankam and P. Picart, "Use of digital color holography for crack investigation in electronic components," Optics Lasers Engineering, 2011, 49(1): 1335-1342.

[37] X. P. Wu, W. R. Gao, and Y. He, "Estimation of parameters for evaluating subsurface microcracks in glass with In-line digital holographic microscopy," Applied Optics, 2016, 55(3): A32-A42.

[38] O. Matoba, H. Inokuchi, K. Nitta, and Y. Awatsuji, "Optical voice recorder by off-axis digital holography," Optics Letters, 2014, 39(22): 6549-6552.

[39] G. U. Kaya and Z. Saraç, "The determination of crack on glass surface by optical voice detector based on digital holography," in Proceeding of 1 st International Conference on Optics, Photonics and Lasers (OPAL' 2018), Barcelona, Spain, 2018, pp. 171-175.

[40] M. Takeda and K. Mutoh, "Fourier transform profilometry for the automatic of 3D object shapes," Applied Optics, 1983, 22(24): 3977-3982. 